\title{
Study on the Target Recognition and Location Technology of industrial Sorting Robot based on Machine Vision
}

\author{
Jiwu Wang ${ }^{1}$ \\ School of Mechanical, Electronic and Control Engineering, Beijing Jiaotong University \\ Beijing 100044, China ${ }^{1}$ \\ Xianwen Zhang ${ }^{1}$, Huazhe Dou ${ }^{1}$ \\ School of Mechanical, Electronic and Control Engineering, Beijing Jiaotong University \\ Beijing 100044, China ${ }^{1}$ \\ Masanori Sugisaka ${ }^{2}$ \\ Alife Robotics Corporation Ltd, Japan and Open University, United Kingdom² \\ E-mail: jwwang@bjtu.edu.cn; ms@alife-robotics.co.jp
}

\begin{abstract}
In order to improve the applications for an industrial sorting robot, it is necessary to increase its flexibility and control accuracy. The prerequisite is to automatically extract the multiple target positions accurately and robustly. The machine vision technology is an effective solution. Here an industrial robot arm is designed and set up for experiment simulation with machine vision. In order to reduce the influence of the size, deformation, and lighting etc., the target recognition and location method with fusion of scale invariant feature transform (SIFT) and moment invariants is developed. The experiments results showed that the developed image processing algorithms are robust, and the flexibility of the industrial robot can be improved by machine vision.
\end{abstract}

Keywords: Arm robot, Target recognition, Flexible control, Machine vision

\section{Introduction}

Intelligent industrial robot, as the concentration and integration of advanced manufacturing technology, information technology and network technology etc., is getting more and more attentation. ${ }^{1}$ This is because a lot of robots and automated equipments will be required in the future applications.

Now, most of the traditional industrial robots can only work according to some fixed paths by an operator's programming. The position of each target is predetermined, and robots only perform repetitive actions. But in the applications of the automated production line or a flexible manufacturing system, ${ }^{2}$ such simple operations are not always satisfied to the practical request. It is necessary to obtain the position of each target automatically, and then adjust the angle of each joint correspondingly. Increasing industrial robot's flexibility and improving its repetitive positioning accuracy is the focus for their further applications. ${ }^{3}$ In order to realize above functions, the prerequisite is to get the target position accurately and reliably. 
Machine vision is a good solution. A CCD camera can provide more information than other sensors. And some pattern recognition algorithms can be used to extract the target and calculate its position automatically in one captured image. Moreover, multiple targets and their positions can be determined at the same time, which makes robots to be flexibly controlled possible.

In order to get the position of each target, the target should be recognized first. Due to the influence of lighting, size, rotation and small scale perspective transformation etc., some target recognition algorithms are not robust. It will cause target positioning unreliable. The SIFT algorithm can effectively improve target recognition capability. In this paper, the SIFT algorithm is used for target recognition, and the target position is calculated with moment invariant method.

\section{Target Recognition Based on Image Matching}

For pattern recognition, some scale and grayscale transformation can not be avoided. It will make some matching algorithm unreliable. The algorithm of the scale invariant feature transform ${ }^{4}$ (SIFT) is a local feature detection algorithm. It is based on matching image feature points to realize target recognition. Even in a complex environment, the target can be quickly and accurately recognized.

The kernel of SIFT algorithm is to search key points in the different scale space, and calculate their direction. The feature points in SIFT are those points, such as corners, edges, dark spot and bright spot etc., which will not be affected by illumination, viewpoint or occlusion. The process of SIFT algorithm is shown in figure 1.

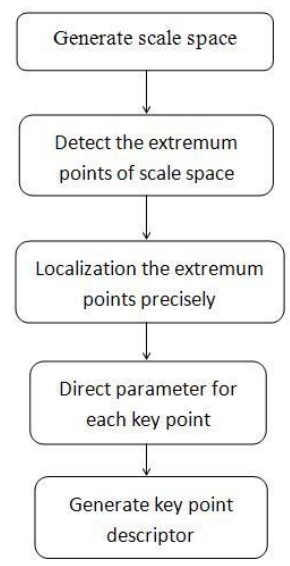

Fig.1.Illustration of SIFT algorithm steps
Image recognition is to identify the target image with the source images in the sample library. Compared with other pattern recognition algorithms, the necessary step is to input the sample target image in the sample library, which simplifies the complex operation for image processing. The target recognition process is shown in figure 2 .

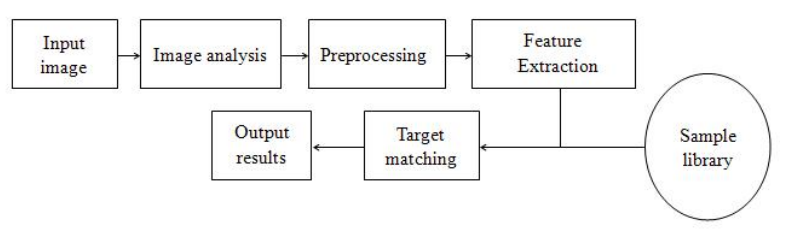

Fig.2. The flow chart of target recognition

In order to verify above algorithms, the following experiments are carried. We take a picture for all sample targets as the sample library, as shown in figure 3. We try to use the targets with different color and shape, even brightness.

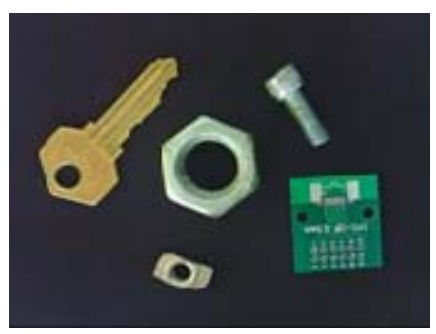

Fig.3. Sample target image library

The matching results are shown in figure 4 .

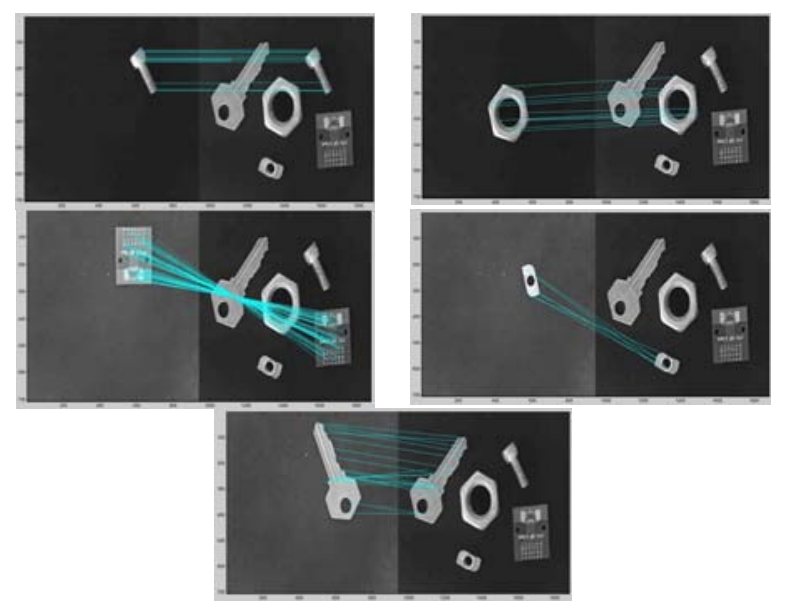

Fig.4 Illustration on the matching results 
In figure 4, the scale and orientation transformation of the target are studied. Moreover, the influence of viewpoint and brightness modification is studied too. As shown in figure 4, with the SIFT algorithm, the matching results are robust and reliable. For each picture in this group, the left side is the input image to be detected, and the right side is the sample library. Their relationship is shown by connecting lines between the extracted feature points.

After the target is extracted by matching with SIFT algorithm, the next step is to determine its position. Generally, the center is necessary for each object, so the moment invariant algorithm is developed. As shown in figure 5, each target area is marked with rectangle, and its center is market with a dot. Their cocodinates are also given in unit of pixel.
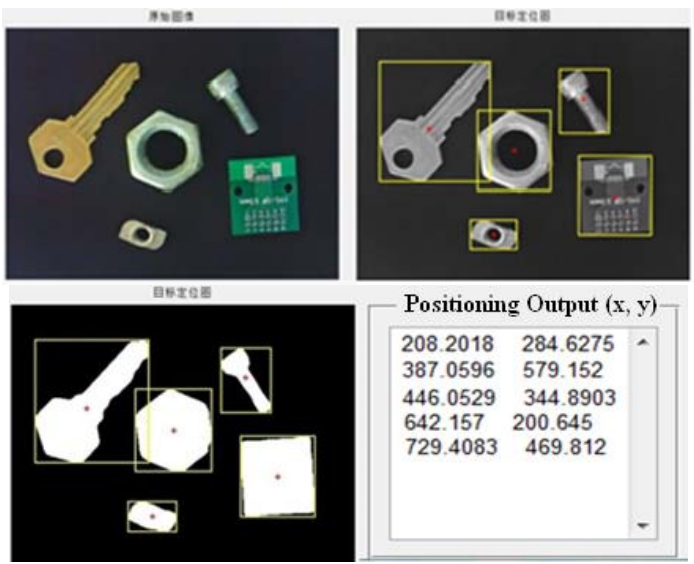

Positioning Output (x, y) $208.2018 \quad 284.6275$ $387.0596 \quad 579.152$ $446.0529 \quad 344.8903$ $642.157 \quad 200.645$ $\begin{array}{lr}729.4083 & 469.812\end{array}$

Fig.5 Illustration on the target positioning

\section{Experiments}

Based on the above target positioning with image processing algorithms of SIFT and moment invariants, the positioning control experiment with our arm robot is carried on. The experiment setting is as shown in figure 6 . The CCD camera is fixed in the arm robot coordinate system. Thus the target position can be easily transformed to the end pose of the manipulator. With inverse kinematics equations, the angle of each joint can be calculated.

The experiment results show that the flexible control for multiple target operations can be performed with image processing methods developed in my lab. Moreover, with SIFTand moment algorithms, the influence of lighting and scale is low to the target position calculation.

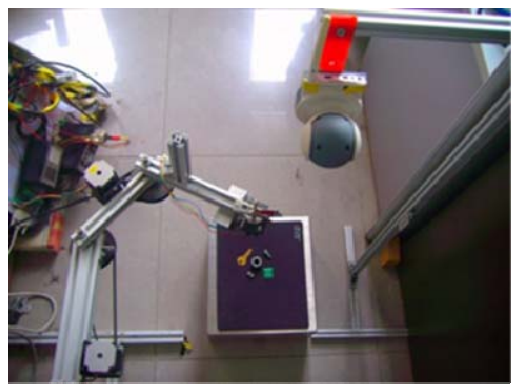

Fig.6.Control experiment with vison matching and positioning

\section{Conclusions}

In this paper, an arm robot and the position control system based on a CCD camera is developed. Multiple target control operations are performed with our lab arm robot. By algorithm fusion of SIFT and moment invariants, the multiple targets can be extracted robust and their positions can be calculated accurately. Moreover, it can effectively control the influence of lighting and scale etc.

\section{References}

1. Y. B. Wang, Y. Z. Hu, and M. Y. Lu, Siemens machine vision system and its application in automobile engine assembly line, Automation systems engineering , vol.1 (2006) 20-22.

2. K. L. Zhuang, J. Z. Wang, and J. Zhou, Application of machine vision in angle inspection, Equipment Manufacturing Technology, vol.4 (2011) 4-10.

3. G. L. David, Distinctive image features from scaleinvariant key points. Int. Journal of Computer Vision, vol.2(60)(2004) 91-110.

4. A. Khotanzad, Y. Huahong, Rotation invariant image recognition using features selected via a systematic method, Pattern recognition, vol.23(10)(1990) 10891101.

5. M. Sugisaka, X. Wang, Intelligent control of a mobile vehicle using on-line learning, System science, vol.25(1999) 41-50.

6. M. Sugisaka, X. Wang, J. Lee, Intelligent control strategy for a mobile vehicle, Applied mathematics and computation, vol.20(1998) 91-98. 